\title{
Gallbladder perforation with concomitant liver abscess: a case report with a review of treatment options
}

\section{Alejandro Quiroga Garza'*, Milton Alberto Muñoz Leija² ${ }^{1 *}$ Juan Manuel Valdivia Balderas $^{1}$, Santos Guzmán López ${ }^{2}$, Rodrigo E. Elizondo Omaña ${ }^{2}$}

\begin{abstract}
${ }^{1}$ Universidad Autonoma de Nuevo Leon, Facultad de Medicina, Human Anatomy Department, Monterrey. Nuevo Leon, Mexico, Instituto Mexicano del Seguro Social, Cirugía General, México

${ }^{2}$ Universidad Autonoma de Nuevo Leon, Facultad de Medicina, Human Anatomy Department, Monterrey. Nuevo Leon, Mexico.
\end{abstract}

Received: 16 January 2020

Revised: 04 March 2020

Accepted: 06 March 2020

\section{*Correspondence:}

Dr. Alejandro Quiroga-Garza,

E-mail: AQuirogaG@gmail.com

Copyright: ( $\subseteq$ the author(s), publisher and licensee Medip Academy. This is an open-access article distributed under the terms of the Creative Commons Attribution Non-Commercial License, which permits unrestricted non-commercial use, distribution, and reproduction in any medium, provided the original work is properly cited.

\section{ABSTRACT}

Gallbladder perforation (GBP) management is still controversial in regards to time (early vs. interval), first procedure (surgical vs. drainage) and technique (laparoscopic vs. open). This is the case report of an adult patient with GBP Niemeier type II, complicated with a concomitant hepatic abscess. The patient was treated medically and with laparoscopic drainage of abscess and pyocholecistolithiasis. She was scheduled shortly after for endoscopic retrograde cholangiopancreatography, and posteriorly for definitive treatment with laparoscopic cholecystectomy 1 month later. Satisfactory postsurgical outcome, with uneventful 1-month follow-up.

Keywords: Case report, Gallbladder perforation, Hepatic abscess, Laparoscopic drainage, Niemeier type II

\section{INTRODUCTION}

Gallbladder (GB) disease is a fairly common pathology. Complications such as spontaneous (non-traumatic) gallbladder perforation (GBP) due to gallstone disease are rare. GBP prevalence ranges from 0.8 to $15 \%$ with a mortality of 12 to $16 \% .^{1-3}$ It is caused by a cascade of events, starting with an obstruction that causes bile stasis, followed by bacterial proliferation, distension, increased pressure, vascular and linfatic collapse, which turns to ischemia, necrosis and finally perforation. ${ }^{2,4}$ The most common site of perforation is the fundus, as it has the lowest vascular supply. ${ }^{5}$

Niemeier introduced a classification for GBP in 1934. Type I for chronic perforation with fistulous communication (cholecystoenteric fistulae); type II for subacute perforation with a surrounding abscess contained by adhesions; and type III for acute perforation and spillage to the cavity with generalized biliary peritonitis. ${ }^{6}$ This classification was later modified by Fletcher and Ravdin in 1952, switching type I and III, without an explanation. This should be referred to as "modified Niemeier classification" although many published articles use the modified classification, referring to and citing the original classification. ${ }^{1-3,6} \mathrm{~A}$ concomitant to GBP is a hepatic abscess formation, in which the visceral wall perforates and expands into the liver. Although rare, this can be classified as a type II GBP, an abscess contained by adhesions or surrounding structures. ${ }^{7}$ Comorbidities such as chronic-degenerative diseases may also complicate patients. ${ }^{1-3}$

Cases are presented with patients suffering from fever, jaundice, upper right quadrant pain and tenderness, and a characteristic palpable mass. Diagnosis is confirmed by 
ultrasound, observing >3 mm wall thickness and sonographic "hole sign". However, visibility may be obstructed by intestinal gas or difficult due to abdominal pain, in which abdominal computed tomography (CT) provides the most sensitive and specific radio-diagnostic tool, also assessing surrounding structures. ${ }^{1-3,8}$

Management is still controversial due to sporadic cases being reported. The debate regarding time (early vs. interval), first procedure (surgical vs. drainage) and technique (laparoscopic vs. open) have yet to be defined. It was discussed the treatment options of an elderly woman with type II GBP, with a concomitant liver abscess.

\section{CASE REPORT}

A 66 year-old woman is evaluated in the emergency room due to unintentional loss of weight, fever, occasional abdominal pain, jaundice, and malaise. The patient has a history of chronic type 2 diabetes mellitus treated with insulin and oral hypoglycemic agents. For several weeks, she had been managed as an out-patient, with a previous abdominal ultrasound reporting a hepatic mass without identifying the GB. Upon examination, there is tenderness and an evident palpable mass in the upper right quadrant. Laboratory results include: white blood cells 14500, hemoglobin 8.7, platelets 569000, glucose $230 \mathrm{mg} / \mathrm{dl}$, creatinine 1.1 , total bilirubin 16.6 , direct bilirubin 11.8, aspartate aminotransferase 224, alanine aminotransferase 189 , prothrombin time 25.4 , partial thromboplastin time 30.7 , international normalized ratio 2.1. An abdominal CT reports air bubbles within a liver abscess with loss of interphase for the GB wall and local inflammation (Figure 1).

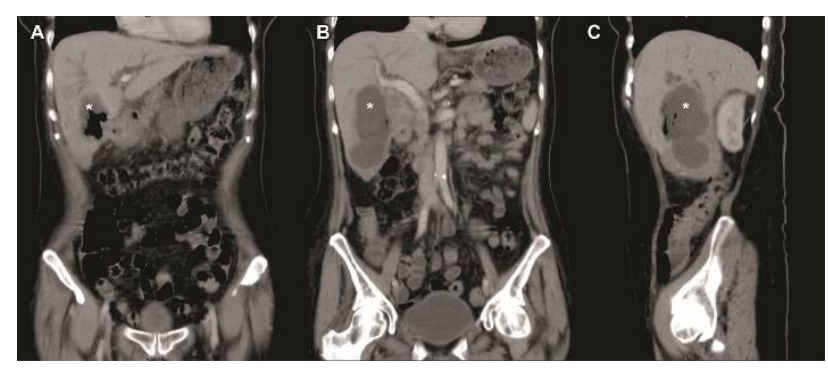

Figure 1: Abdominal computed tomography of the patient with gallbladder perforation and hepatic abscess. a. coronal image evidencing the presence of air within the hepatic abscess. The gallbladder is not clearly identified, and there are adhesions of the pyloric antrum to the local inflammation. b. a coronal image shows the extension of the abscess to segments $\mathrm{V}$ and VI. c. para-sagittal image of hepatic abscess in segment VI. *: hepatic abscess

Image-guided drainage was proposed as the first option, but is not available at the hospital. The patient is scheduled for emergency laparoscopy, in which GBP Niemeier type II is identified due to a severe (grade III) cholecystitis. ${ }^{6,9}$ There is abundant local inflammation with firm adhesions of the stomach, duodenum, and omentum to the GB. Dissection of the fundus provides access to the interior of the GB and liver abscess, draining necrotic tissue and abundant pus that extends primarily into hepatic segments V and VI (Figure 2).

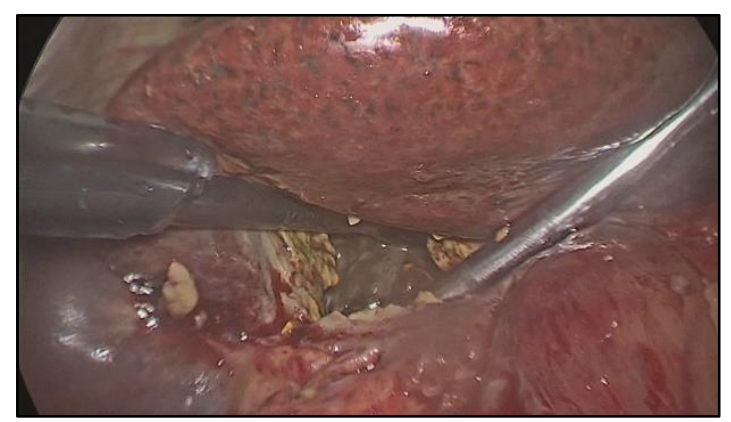

Figure 2: Laparoscopic view of gallbladder surgical opening for drainage. Local anatomy inflammation did not permit dissection.

Local anatomy is distorted, and structures cannot be clearly identified. In accordance with the TG13: Updated Tokyo guidelines for cholecystitis, 9 the surgeon decides to place drainage in the GB and abscess, continue medical treatment, improve patient's general condition, and schedule a delayed cholecystectomy. She is transferred to the hepatic-biliary unit in another hospital. Five days later, endoscopic retrograde cholangiopancreatography (ERCP) drainage is performed, and total parental nutrition is begun due to torpid evolution, low tolerance to oral intake and low mobility. One month later, successful subtotal laparoscopic cholecystectomy is performed. The patient developed left pleural effusion, managed medically. Follow-up continued uneventfully at 1-month.

\section{DISCUSSION}

It is a rare entity for a GBP to sealed with the stomach and duodenum, and continue with perforation into the liver, creating an abscess. Fistula and abscess are most common amongst younger patients, while biliary peritonitis is most frequent in the elderly.2 Management of any type of GBP is not well defined in the literature, finding scarce case reports with different surgical options. TG13 Tokyo guidelines do not directly address GBP, although severe cholecystitis (grade III) is recommended to be managed with drainage and delayed elective cholecystectomy. ${ }^{2,7,9}$

Antibiotic and anti-inflammatory therapy is essential in the patient's treatment. Most authors seem to agree percutaneous drainage (either ultrasound or CT guided) is the preferred first-choice treatment to improve conditions. ${ }^{2,3}$ This has been reported to be effective in resolving 70 to $90 \%$ of intrahepatic abscesses.3 Although some authors omit this step, cholecystectomy is established as the definitive treatment. Surgery in early 
vs. interval time, and conventional/open vs. laparoscopic technique is still highly debated. ${ }^{1,3,7-9}$ Many authors debate the procedure should be started as minimally invasive (laparoscopic) due to the better view, and access to remanent liver abscess drainage, however, a high rate of conversion is reported. ${ }^{3,7-8}$ Regardless of the technique, the most important step of any cholecystectomy, is, without doubt, the direct visualization of the anatomy and critical view of safety (cystic duct and cystic artery). Failure to create this view is an indication of subtotal cholecystectomy or conversion to open cholecystictomy. ${ }^{7,9,10}$ Derici et al retrospectively reviewed their cases of GBP, reporting early surgical treatment in all their patients although less than a third had been correctly diagnosed with GBP prior to the surgical intervention. Their mean hospital stay was 15 days, with a general mortality of $12.5 \% .^{1}$ Comorbidities and other factors have an influence on all of this. Patients with gallbladder stones, malignancies, trauma, corticosteroid or immunosuppression therapy, diabetes, old age, and male gender have a higher probability of complications. ${ }^{1-3}$

Due to lack of resources, as is many times the case in middle and low-income countries, our patient was managed with laparoscopic drainage, instead of percutaneous. Although this allowed better visualization of the pathology, the patient was exposed to general anesthesia. This is a limitation on patient management, but it controlled the abscess and allowed for patient improvement. The authors recommend percutaneous drainage over laparoscopic, if available.

Physicians are taught to make evidence-based medicine, however, rare cases and uncertainty are what promote clinical reasoning to make the best possible decisions for their patients, after evaluating all aspects of a case. ${ }^{11}$ There are few reports of liver abscess related to gallbladder perforation, and its management is not well established.

Case reports and GB disease management guidelines tend to recommend drainage and subsequently, cholecystectomy, either laparoscopic or open, as the best treatment. A meta-analysis of case reports and case series is needed to establish a standard treatment to include in future revisions of Tokyo guidelines and recommendations, addressing GBP pathology specifically.

\section{CONCLUSION}

GBP Niemeier type II management is not well defined by guidelines and literature. Percutaneous drainage and subsequent laparoscopic cholecystectomy have the best evidence. However, a meta-analysis is needed to confirm the level of evidence.
Funding: No funding sources

Conflict of interest: None declared

Ethical approval: Not required

\section{REFERENCES}

1. Derici H, Kara C, Bozdag AD, Nazli O, Tansug T, Akca E. Diagnosis and treatment of gallbladder perforation. World J Gastroenterol. 2006;12(48):7832-6.

2. Hussain T, Adams M, Ahmed M, Arshad N, Solkar M. Intrahepatic perforation of the gallbladder causing liver abscesses: case studies and literature review of a rare complication. Ann R Coll Surg Engl. 2016;98(6):88-91

3. Kochar K, Vallance K, Mathew G, Jadhav V. Intrahepatic perforation of the gall bladder presenting as liver abscess: case report, review of literature and Niemeier's classification. Eur $\mathbf{J}$ Gastroenterol Hepatol. 2008;20(3):240-4.

4. Taneja S, Sharma A, Duseja AK, Kalra N, Chawla Y. Spontaneous perforation of gallbladder with intrahepatic bilioma. J Clin Exp Hepatol. 2011;1:210-1.

5. Morris BS, Balpande PR, Morani AC, Chaudhary RK, Maheshwari M, Raut AA. The CT appearances of gallbladder perforation. $\mathrm{Br} \mathrm{J}$ Radiol. 2007;80:898-901.

6. Niemeier OW. Acute free perforation of the gallbladder. Ann Surg. 1934;99:922-4.

7. Nikumbh T, Bhandarwar A, Sanap S, Wagholikar G. Laparoscopic management of intra-hepatic gallbladder perforation. J Minim Access Surg. 2020;16(1):77-9.

8. Singh K, Singh A, Vidyarthi SH, Jindal S, Thounaojam CK. Spontaneous intrahepatic type II gallbladder perforation: a rare cause of liver abscess - case report. J Clin Diagn Res. 2013;7:2,12-4.

9. Yamashita Y, Takada T, Strasberg SM, Pitt HA, Gouma DJ, Garden OJ, et al. TG13 surgical management of acute cholecystitis. J Hepatobiliary Pancreat Sci. 2013;20(1):89-96.

10. Elshaer M, Gravante G, Thomas K, Sorge R, AlHamali S, Ebdewi H. Subtotal cholecystectomy for "difficult gallbladders": systematic review and meta-analysis. JAMA Surg. 2015;150(2):159-68.

11. Garza QA, Garza TR, Omaña RE, López GS. The Use of clinical reasoning skills in the setting of uncertainty: a case of trial femoral head migration. Anat Sci Educ. 2019;10:1002.

Cite this article as: Quiroga-Garza A, Muñoz-Leija MA, Valdivia-Balderas JM, Guzmán-López S, Elizondo-Omaña RE. Gallbladder perforation with concomitant liver abscess: a case report with a review of treatment options. Int Surg J 2020;7:1283-5. 